\title{
A Fourth-Order Collocation Scheme for Two-Point Interface Boundary Value Problems
}

\author{
Rakhim Aitbayev ${ }^{1}$ and Nazgul Yergaliyeva ${ }^{2}$ \\ ${ }^{1}$ Department of Mathematics, New Mexico Institute of Mining and Technology, Socorro, NM 87801, USA \\ ${ }^{2}$ Department of Information Technology, Kazakh National Technical University, 22 Satpayev Street, Almaty 050013, Kazakhstan \\ Correspondence should be addressed to Rakhim Aitbayev; aitbayev@nmt.edu
}

Received 7 August 2014; Accepted 9 November 2014; Published 24 November 2014

Academic Editor: Weimin Han

Copyright (c) 2014 R. Aitbayev and N. Yergaliyeva. This is an open access article distributed under the Creative Commons Attribution License, which permits unrestricted use, distribution, and reproduction in any medium, provided the original work is properly cited.

\begin{abstract}
A fourth-order accurate orthogonal spline collocation scheme is formulated to approximate linear two-point boundary value problems with interface conditions. The coefficients of the differential operator may have jump discontinuities at the interface point, a nodal point of the scheme. Existence and uniqueness of the numerical solution are proved. Optimal order error estimates in the maximum norm are obtained, and a superconvergence property of the numerical solution in the maximal nodal norm is proved. Numerical results are presented confirming the theoretical estimates.
\end{abstract}

\section{Introduction}

In this paper, we propose and analyse for existence, uniqueness, and convergence an orthogonal spline collocation (OSC) scheme for the numerical solution of a linear twopoint boundary value problem (BVP) with two additional interface conditions. The variable coefficients of the differential equation may have jump discontinuities at the interface point. Existence and uniqueness of the BVP were studied in [1].

The interface boundary value problems (IBVPs) are also known as diffraction problems or BVPs with transmission (transmittal) conditions. In one dimension, they may be viewed as multipoint BVPs with piecewise continuous coefficients. IBVPs originate, for example, in heat or mass transfer in composite materials or materials with thin porous barriers, elasticity of heterogeneous materials, and population genetics $[2,3]$. In the case of heat transfer in layered composite materials, the temperature is discontinuous across the interface whereas the heat flux is continuous. The temperature difference is proportional to the heat flux, and the proportionality constant is known as the interfacial thermal (contact) resistance.
IBVPs can be solved numerically by, for example, homogeneous finite difference schemes [4], the shooting method [5], the immersed boundary method [6], the immersed interface method [7-9], or the ghost fluid method [10]. A fourthorder finite element method with Hermite elements for onedimensional interface problems is studied in [11]. By changing variables, the problem can be transformed to a regular twopoint BVP for a system of differential equations [12].

In this work, the OSC method is applied with the finite element space of piecewise Hermite cubic functions, and the interface conditions are incorporated into the scheme directly. The assumption that an interface point is a grid point restricts straight forward generalization of the proposed scheme on two-dimensional problems with a curved interface. Nevertheless, the proposed scheme is a simple modification of the OSC method for a two-point BVP; it exhibits optimal fourth-order convergence, and it is simple to implement. The interface conditions are explicitly put into the stiffness matrix, and this does not change the almost block diagonal structure of the matrix of the standard, noninterface BVP. For other numerical methods for two-point IBVPs, it might not be this easy to incorporate such general interface conditions into the stiffness matrix. 
The OSC method is a collocation scheme where collocation points are the nodes of the composite Gaussian quadrature. A survey of the OSC method for solving onedimensional BVPs is given in [13]. The numerical solution is sought in a space of smooth piecewise polynomial functions. The main advantages of the OSC method are a simple formulation, a relatively easy implementation, optimal order error estimates, and the so-called superconvergence property at the nodal points.

Schwarz and de Boor proposed the OSC method in [14], where, for an $m$ th order nonlinear two-point BVP, they proved existence and uniqueness of the numerical solution and obtained the maximum norm and the maximal nodal norm convergence estimates. In [15], the authors briefly noted (see the second paragraph on page 26) that their analysis can be extended to more general problems in which differential operators may have different orders on different subintervals of the domain with appropriate side conditions at the end points of the subintervals, that is, to IBVPs. In this work, we applied that idea to linear IBVPs with general interface conditions. We follow the methodology of $[14,15]$, and the key elements of the analysis are existence and regularity of Green's function and approximation properties of the socalled "ssuper" projectors named in honor of S. A. Pruess, who was the first to consider such projectors (the word "ssuper" is a permutation of letters in "Pruess"). The case of one interface point is studied for simplicity of presentation. The obtained results can be generalized in a straightforward manner to problems with many interface points.

The outline of the paper is as follows. In Section 2, we formulate IBVP, introduce notation, and rewrite the problem as an integral equation. In Section 3, we introduce the OSC scheme and obtain its algebraic form for a finite element basis. In Section 4, we analyze the scheme for existence and uniqueness and obtain error estimates. In Section 5, we present two numerical examples, and, in Section 6, we give our concluding remarks.

\section{Problem Formulation}

For an integer $k \geq 0$, let $C^{k}\left[t_{1}, t_{2}\right]$ be the vector space of $k$ times continuously differentiable functions with the standard norm $\|\cdot\|_{C^{k}\left[t_{1}, t_{2}\right]}$. Let $(a, b)$ be a line interval, and let $c \in(a, b)$. Let $Q^{k}$ be the vector space of functions $v$ defined on $[a, c] \cup$ $[c, b]$ such that

$$
\left.v\right|_{[a, c]} \in C^{k}[a, c],\left.\quad v\right|_{[c, b]} \in C^{k}[c, b] .
$$

Note that $v$ and its derivatives can only have jump discontinuities at the interface point $x=c$, where $v$ may be doublevalued. Let

$$
v^{ \pm}=\lim _{x \rightarrow c \pm} v(x), \quad[v]=v^{+}-v^{-} .
$$

Let

$$
\|v\|_{k}=\max \left\{\|v\|_{C^{k}[a, c]},\|v\|_{C^{k}[c, b]}\right\} .
$$

Denote $D^{k}=d^{k} / d x^{k}$ and $\partial_{x}^{k}=\partial^{k} / \partial x^{k}$.
In this paper, we consider the differential equation

$$
L u \equiv-u^{\prime \prime}+p u^{\prime}+q u=f \quad \text { in }(a, c) \cup(c, b),
$$

subject to the boundary conditions

$$
\begin{aligned}
& l_{a}(u) \equiv a_{0} u(a)-a_{1} u^{\prime}(a)=\alpha, \\
& l_{b}(u) \equiv b_{0} u(b)+b_{1} u^{\prime}(b)=\beta,
\end{aligned}
$$

and the additional general linear interface conditions

$$
l_{i}(u) \equiv c_{i, 1} u^{-}+c_{i, 2}\left(u^{\prime}\right)^{-}+c_{i, 3} u^{+}+c_{i, 4}\left(u^{\prime}\right)^{+}=\gamma_{i}, \quad i=1,2 .
$$

Functions $p, q$, and $f$ belong to $Q^{0}$, and $a_{j}, b_{j}, c_{i j}, \gamma_{i}, \alpha$, and $\beta$ are reals.

For example, consider the differential equation

$$
-\left(k u^{\prime}\right)^{\prime}+q u=f \quad \text { in }(a, c) \cup(c, b),
$$

with the discontinuous coefficients $k, q \in Q^{0}$, subject to the boundary conditions ( $4 \mathrm{~b}$ ) and one of the following common types of interface conditions: (1) the continuity (perfect contact) interface conditions: $[u]=0,\left[k u^{\prime}\right]=0$; (2) flux jump interface conditions: $[u]=0,\left[k u^{\prime}\right]=d_{0} u(c), d_{0}>0$; (3) a linear radiation (a thermal contact resistance) condition: $h[u]=k u^{\prime},\left[k u^{\prime}\right]=0, h>0$.

In what follows, we assume that problem (4a)-(4c) has a unique solution. Let us list some of the properties of Green's function $G(x, s)$ of the problem (see $[1,16])$. Green's function $G$ is continuous in the square $[a, b]^{2}$ everywhere except the lines $x=c$ and $s=c$, where it may have finite discontinuities. For a fixed $s \in[a, b], G$ satisfies the differential equation (4a) almost everywhere on $(a, b)$ and the homogeneous boundary and the interface equations (4b) and (4c). The derivative $\partial G / \partial x$ has, in addition, the jump discontinuity across the line $x=s$.

The lines $x=c, s=c$, and $x=s$ divide domain $[a, c]^{2}$ into 4 triangular and 2 rectangular closed subregions. Let $\mathscr{D}$ be the set consisting of these 6 subregions. For a function $\widetilde{G}(x, s)$ defined and sufficiently smooth in $[a, b]^{2}$ and nonnegative integers $r$ and $k$, let

$$
\|\widetilde{G}\|_{r, k}=\max _{i \leq r} \max _{j \leq k} \max _{D \in \mathscr{D}}\left\|\partial_{x}^{i} \partial_{s}^{j} \widetilde{G}\right\|_{C(D)} .
$$

For any integer $k \geq 0$, Green's function $G$ is in $C^{k+2}$ on every subregion in $\mathscr{D}$ provided that the coefficients $p$ and $q$ are in $Q^{k}$. In particular, if $p, q \in Q^{0}$, then

$$
\|G\|_{2,2}<+\infty \text {. }
$$

In our analysis, we make use of an integral form of IBVP (4a)-(4c). Define a nonlinear operator $T: L^{1}(a, b) \rightarrow Q^{0}$ as follows. For any $g \in L^{1}(a, b)$, let $U \in Q^{1}$ be the unique solution of the problem

$$
\begin{gathered}
U^{\prime \prime}=g \quad \text { in }(a, c) \cup(c, b), \\
l_{a}(U)=\alpha, \quad l_{b}(U)=\beta, \\
l_{i}(U)=\gamma_{i}, \quad i=1,2 .
\end{gathered}
$$


Let

$$
T(g)=p U^{\prime}+q U-f .
$$

IBVP (4a)-(4c) is equivalent to the following integral equation: find $y \in Q^{0}$ such that

$$
y=T(y) .
$$

Indeed, if $y$ is the solution of (10) and $u$ is the solution of (8) with $g=y$, then $u^{\prime \prime}=p u^{\prime}+q u-f$.

Let $G_{0}$ be Green's function of problem (8). If $u_{0} \in Q^{0}$ is the piecewise linear function that satisfies conditions $(4 \mathrm{~b})$ and (4c), then the solution $U$ of problem (8) can be written in the form

$$
U(x)=u_{0}(x)+\int_{a}^{b} G_{0}(x, s) g(s) d s .
$$

Using (9) and (11), operator $T$ can be written in the form

$$
\begin{aligned}
T(g)= & p u_{0}^{\prime}+q u_{0}-f+p \int_{a}^{b} \partial_{x} G_{0}(\cdot, s) g(s) d s \\
& +q \int_{a}^{b} G_{0}(\cdot, s) g(s) d s .
\end{aligned}
$$

The formal derivative of $T$ acting on function $g$ is

$$
T^{\prime}(g)=\int_{a}^{b}\left(p(\cdot) \partial_{x} G_{0}(\cdot, s)+q(\cdot) G_{0}(\cdot, s)\right) g(s) d s .
$$

\section{OSC Scheme}

For an integer $N>0$, let $\pi=\left\{x_{i}\right\}_{i=0}^{N}$ be a regular partition of the interval $[a, b]$ such that

$$
a=x_{0}<x_{1}<\cdots<x_{N}=b,
$$

and let the interface point $x=c$ be an interior partition knot with the index $i_{c}$. Let $I_{i}=\left[x_{i-1}, x_{i}\right], h_{i}=x_{i}-x_{i-1}$, and $h=$ $\max \left\{h_{i}\right\}$. Let $\mathscr{G}_{h}=\left\{\xi_{i, 1}, \xi_{i, 2}\right\}_{i=1}^{N}$ be the sequence of the Gauss points in interval $[a, b]$ corresponding to partition $\pi$; that is,

$$
\xi_{i, 1}=x_{i-1}+\frac{3-\sqrt{3}}{6} h_{i}, \quad \xi_{i, 2}=x_{i-1}+\frac{3+\sqrt{3}}{6} h_{i} .
$$

Let $C_{\pi}$ be the vector space of functions defined on $\bigcup_{i=1}^{N} I_{i}$ and continuous on every subinterval $I_{i}$. Functions in $C_{\pi}$ can be double-valued at the knots of $\pi$. Let

$$
\|v\|=\max \left\{\|v\|_{C\left(I_{i}\right)}: 1 \leq i \leq N\right\} .
$$

For an integer $k \geq 0$, let $P_{k, \pi}$ be a subspace of $C_{\pi}$ consisting of piecewise polynomial functions such that every $p \in P_{k, \pi}$ restricted on every subinterval $I_{i}$ is a polynomial of degree $k$.

Let $\widehat{V}_{h}=C^{1}[a, b] \cap P_{3, \pi}$ be the space of piecewise Hermite cubic polynomials [17, Chapter 3$]$, and let

$$
V_{h}=Q^{1} \cap P_{3, \pi}
$$

be a vector space of modified piecewise Hermite cubic functions that may be discontinuous at the interface knot $x_{i}$. A piecewise cubic function $v$ in $V_{h}$ is continuously differentiable on $[a, c]$ and $[c, b]$, and $v$ can be double-valued at the interface knot $x_{i_{c}}$. We approximate IBVP (4a)-(4c) by the following OSC scheme: find $u_{h} \in V_{h}$ such that

$$
L u_{h}(\xi)=f(\xi) \quad \forall \xi \in \mathscr{G}_{h},
$$

and $u_{h}$ satisfies the boundary conditions ( $4 \mathrm{~b}$ ) and the interface conditions (4c).

To obtain an algebraic form of the OSC problem, we introduce a Hermite type finite element basis in $V_{h}$. For $i=$ $0, \ldots, N$, define the value and the slope basis functions $v_{i}$ and $s_{i}$ in $\widehat{V}_{h}$, respectively, by

$$
\begin{array}{ll}
v_{i}\left(x_{j}\right)=\delta_{i j}, & v_{i}^{\prime}\left(x_{j}\right)=0, \\
s_{i}\left(x_{j}\right)=0, & s_{i}^{\prime}\left(x_{j}\right)=\delta_{i j}, \\
& j=0, \ldots, N .
\end{array}
$$

The sequence $\left\{v_{i}, s_{i}\right\}_{i=0}^{N}$ is a basis for $\widehat{V}_{h}$. We modify this set to a basis for $V_{h}$. Define discontinuous value basis functions

$$
\begin{aligned}
& v_{i_{c}}^{l}(x)= \begin{cases}v_{i_{c}}(x), & x \in[a, c], \\
0, & x \in[c, b],\end{cases} \\
& v_{i_{c}}^{r}(x)= \begin{cases}0, & x \in[a, c], \\
v_{i_{c}}(x), & x \in[c, b],\end{cases}
\end{aligned}
$$

and, similarly, define discontinuous slope basis functions $s_{i_{c}}^{l}$ and $s_{i_{c}}^{r}$. The sequence

$$
\begin{gathered}
\beta=\left\{v_{0}, s_{0}, \ldots, v_{i_{c}-1}, s_{i_{c}-1}, v_{i_{c}}^{l}, s_{i_{c}}^{l}, v_{i_{c}}^{r},\right. \\
\left.s_{i_{c}}^{r}, v_{i_{c}+1}, s_{i_{c}+1}, \ldots, v_{N}, s_{N}\right\}
\end{gathered}
$$

is a finite element basis for $V_{h}$. Let $\beta=\left\{\phi_{1}, \ldots, \phi_{M}\right\}$, where $M=\operatorname{dim}\left(V_{h}\right)=\operatorname{dim}\left(\widehat{V}_{h}\right)+2=2 N+4$. The solution of the OSC problem (18) can be written in the form $u_{h}(x)=\sum_{i=1}^{M} u_{i} \phi_{i}(x)$ with the associated solution vector $\mathbf{u}=$ $\left(u_{1}, \ldots, u_{M}\right)^{T}$.

Let $C=\left(c_{i j}\right) \in R^{2 \times 4}$ be the matrix corresponding to the interface conditions (4c), and let, for $i=1, \ldots, N$,

$$
A_{i}=\left(\begin{array}{llll}
L v_{i-1}\left(\xi_{i, 1}\right) & L s_{i-1}\left(\xi_{i, 1}\right) & L v_{i}\left(\xi_{i, 1}\right) & L s_{i}\left(\xi_{i, 1}\right) \\
L v_{i-1}\left(\xi_{i, 2}\right) & L s_{i-1}\left(\xi_{i, 2}\right) & L v_{i}\left(\xi_{i, 2}\right) & L s_{i}\left(\xi_{i, 2}\right)
\end{array}\right) .
$$

Similarly, define matrices $A_{i_{c}}^{\prime}$ and $A_{i_{c}+1}^{\prime}$ by replacing functions with index $i_{c}$ in $A_{i_{c}}$ and $A_{i_{c}+1}$ by the corresponding discontinuous basis functions. Let

$$
\begin{aligned}
\widetilde{A}=\operatorname{diag}( & A_{1}, \ldots, A_{i_{c}-1}, A_{i_{c}}^{\prime}, C, A_{i_{c}+1}^{\prime}, \\
\left.A_{i_{c}+2}, \ldots, A_{N}\right) & \in R^{(2 N+2) \times(2 N+4),},
\end{aligned}
$$

and note that it is a block diagonal matrix whose every two consecutive $2 \times 4$ diagonal blocks have the two-column 
overlap. Finally, let $A \in R^{(2 N+4) \times(2 N+4)}$ be the matrix obtained by augmenting matrix $\widetilde{A}$ with the rows

$$
\left(a_{0},-a_{1}, 0, \ldots, 0\right), \quad\left(0, \ldots, 0, b_{0}, b_{1}\right)
$$

on top and bottom, respectively, where $a_{i}$ and $b_{i}$ are the coefficients of the boundary conditions (4b). Matrix $A$ has an almost block diagonal structure described in [18]. Note that the $2 \times 4$ interface condition matrix $C$ naturally "fits" into the matrix and does not change its structure. Let

$$
\begin{gathered}
\mathbf{f}=\left(\gamma_{a}, \bar{f}_{1}, \ldots, \bar{f}_{i_{c}-1}, \gamma_{1}, \gamma_{2}, \bar{f}_{i_{c}+1}, \ldots, \bar{f}_{N}, \gamma_{b}\right)^{T}, \\
\bar{f}_{i}=\left(f\left(\xi_{i, 1}\right), f\left(\xi_{i, 2}\right)\right),
\end{gathered}
$$

be the right hand side vector. The OSC problem (18) can be written in the algebraic form

$$
A \mathbf{u}=\mathbf{f}
$$

The blocks of $\widetilde{A}$ can be computed using the representation

$$
A_{i}=-B_{i}^{(2)}+P_{i} B_{i}^{(1)}+Q_{i} B_{i}^{(0)}
$$

where $P_{i}=\operatorname{diag}\left(p\left(\xi_{i, 1}\right), p\left(\xi_{i, 2}\right)\right)$ and $Q_{i}=\operatorname{diag}\left(q\left(\xi_{i, 1}\right), q\left(\xi_{i, 2}\right)\right)$, and

$$
B_{i}^{(l)}=\left[\begin{array}{llll}
v_{i-1}^{(l)}\left(\xi_{i, 1}\right) & s_{i-1}^{(l)}\left(\xi_{i, 1}\right) & v_{i}^{(l)}\left(\xi_{i, 1}\right) & s_{i}^{(l)}\left(\xi_{i, 1}\right) \\
v_{i-1}^{(l)}\left(\xi_{i, 2}\right) & s_{i-1}^{(l)}\left(\xi_{i, 2}\right) & v_{i}^{(l)}\left(\xi_{i, 2}\right) & s_{i}^{(l)}\left(\xi_{i, 2}\right)
\end{array}\right],
$$

where $v^{(l)}=D^{l} v$.

\section{Convergence Analysis}

In the sequel, $C$ is a generic positive constant independent of partition $\pi$. The following is the main result of this paper on existence, uniqueness, and convergence of the OSC scheme (18).

Theorem 1. Let $p, q$, and $f$ be in $Q^{0}$, let $u \in Q^{2}$ be a unique solution of IBVP (4a)-(4c), and let $G$ be Green's function of the problem.

(1) The OSC problem (18) has a unique solution $u_{h} \in V_{h}$.

(2) If $p, q, f \in Q^{n}, n \leq 2$, then

$$
\left\|D^{r}\left(u-u_{h}\right)\right\| \leq C h^{\min \{n, 2\}}, \quad r \leq 2 .
$$

(3) If $p, q, f \in Q^{2+n}$, then,

(a) for $r \leq 1$ and $n \leq 2$,

$$
\max _{x \in \pi}\left|D^{r} u(x)-D^{r} u_{h}(x)\right| \leq C h^{2+n} \psi_{n, r},
$$

(b) for $n \leq 2$ if $r=0$, and for $n \leq 1$ if $r=1$,

$$
\left\|D^{r}\left(u-u_{h}\right)\right\| \leq C h^{2+n} \psi_{n, r},
$$

$$
\begin{aligned}
& \text { where } \\
& \psi_{n, r}=\left\|\partial_{x}^{r} G\right\|_{0, n}\|u\|_{4+n}\left(1+\|p\|_{2+n}+\|q\|_{2+n}\right) .
\end{aligned}
$$

Inequalities in (30) are the maximal nodal norm error estimates. Note that the convergence is of order 4 for the derivative $u_{h}^{\prime}$ if $u \in Q^{6}$; this is known as the superconvergence property of the OSC solution. Inequalities in (29) and (31) are the maximum norm global error estimates; they imply

$$
\left\|D^{k}\left(u-u_{h}\right)\right\| \leq C h^{4-k}\|u\|_{6-k}, \quad k=0,1,2,
$$

which are optimal order error estimates. The estimate for $u_{h}^{\prime \prime}$ is also optimal by regularity, whereas estimates for $u_{h}$ and $u_{h}^{\prime}$ are not. These results are typical for the OSC method [13].

To prove Theorem 1, we use the approach and results in $[14,15]$. For a given $g_{h} \in P_{1, \pi}$, consider the differential equation

$$
U_{h}^{\prime \prime}=g_{h}
$$

subject to the interface and the boundary conditions (4c) and (4b), respectively. Using (11) and (9), get

$$
\begin{gathered}
U_{h}=u_{0}+\int_{a}^{b} G_{0}(x, s) g_{h}(s) d s, \\
T\left(g_{h}\right)=p U_{h}^{\prime}+q U_{h}-f .
\end{gathered}
$$

The OSC problem (18) can be written in an equivalent integral form: find $y_{h} \in P_{1, \pi}$ such that

$$
y_{h}(\xi)=\left(T\left(y_{h}\right)\right)(\xi), \quad \forall \xi \in \mathscr{G}_{h} .
$$

First, we prove two auxiliary lemmas. The following statement follows from Lemma 3.1 in [14].

Lemma 2. Assume that functions $p, q$, and $f$ are in $Q^{0}$, and let $y \in Q^{0}$ be the unique solution of problem (10). Then the OSC problem (36) has a unique solution $y_{h}$, and

$$
\left\|y-y_{h}\right\| \leq C \sup \left\{\|y-\tilde{p}\|: \tilde{p} \in P_{1, \pi}\right\} .
$$

If $y \in Q^{n}, n \leq 2$, then

$$
\left\|y-y_{h}\right\| \leq C h^{\min \{n, 2\}}
$$

Proof. Let $T: P_{1, \pi} \rightarrow Q^{0}$ be defined by (12), and let $T^{\prime}$ be its Fréchet derivative given by (13). First note that the statement of Lemma 3.1 in [14] remains valid with space $C[a, b]$ replaced by $Q^{0}$, that is, replacing $C[a, b]$ in the assumptions $y \in C[a, b]$ and $T^{\prime} v \in C[a, b]$ by $Q^{0}$ and replacing the modulus of continuity $\omega_{v}$ by the function

$$
\widetilde{\omega}_{v}(\delta)=\max \left\{\omega_{v,[a, c]}(\delta), \omega_{v,[c, b]}(\delta)\right\}, \quad v \in Q^{0},
$$

where $\omega_{v, I}$ is the modulus of continuity of $v$ on the closed interval $I$. Then an auxiliary inequality (2.1) in [14] holds over $Q^{0}$. 
Let us now prove that the assumptions of Lemma 3.1 of [14] are satisfied by operators $T$ and $T^{\prime}$ defined by (12) and (13). Since

$$
E(v) \equiv T(v)-T(y)-T^{\prime}(v-y)=0,
$$

assumption (i) of Lemma 3.1 of [14] is trivially satisfied.

Assumption (ii) requires that, for every $v \in C_{\pi}, T^{\prime}(v) \in$ $Q^{0}$, and

$$
\widetilde{\omega}_{T^{\prime}(v)} \leq\|v\| \omega
$$

for some nondecreasing subadditive function $\omega(\delta) \epsilon$ $C[0, \min \{b-c, c-a\}]$ independent of $\pi$ and $v$ such that $\lim _{\delta \rightarrow 0} \omega(\delta)=0$. Note that $T^{\prime}(v) \in Q^{0}$ by its definition (13) and properties of Green's function $G_{0}$.

Let $a \leq x_{1}<x_{2} \leq c$ and $r \leq 1$, and obtain

$$
\begin{aligned}
& \int_{a}^{b}\left|\partial_{x}^{r}\left(G_{0}\left(x_{1}, s\right)-G_{0}\left(x_{2}, s\right)\right)\right| d s \\
& =\int_{a}^{x_{1}}+\int_{x_{1}}^{x_{2}}+\int_{x_{2}}^{c}+\int_{c}^{b} \\
& \leq\left[\left(x_{1}-a\right)+\left(c-x_{1}\right)+(b-c)\right]\left\|G_{0}\right\|_{r+1,0}\left|x_{1}-x_{2}\right| \\
& \quad+2\left\|G_{0}\right\|_{r, 0}\left|x_{1}-x_{2}\right| \\
& \quad \leq \max \{2, b-a\}\left\|G_{0}\right\|_{2,0}\left|x_{1}-x_{2}\right| .
\end{aligned}
$$

The same estimate holds for $c \leq x_{1}<x_{2} \leq b$.

Now let $v \in C_{\pi}$. For any $x_{1}$ and $x_{2}$ in $[a, c]$ or $[c, b]$, using (42), obtain

$$
\begin{aligned}
\mid T^{\prime}(v) & \left(x_{1}\right)-T^{\prime}(v)\left(x_{2}\right) \mid \\
\leq & \|v\| \int_{a}^{b}\left|p\left(x_{1}\right) \partial_{x} G_{0}\left(x_{1}, s\right)-p\left(x_{2}\right) \partial_{x} G_{0}\left(x_{2}, s\right)\right| d s \\
& +\|v\| \int_{a}^{b}\left|q\left(x_{1}\right) G_{0}\left(x_{1}, s\right)-q\left(x_{2}\right) G_{0}\left(x_{2}, s\right)\right| d s \\
\leq & \|v\|\|p\|_{0} \int_{a}^{b}\left|\partial_{x} G_{0}\left(x_{1}, s\right)-\partial_{x} G_{0}\left(x_{2}, s\right)\right| d s \\
& +\left\|G_{0}\right\|_{1,0}\|v\| \widetilde{\omega}_{p}\left(\left|x_{1}-x_{2}\right|\right) \\
& +\|v\|\|q\|_{0} \int_{a}^{b}\left|G_{0}\left(x_{1}, s\right)-G_{0}\left(x_{2}, s\right)\right| d s \\
& +\left\|G_{0}\right\|_{1,0}\|v\| \widetilde{\omega}_{q}\left(\left|x_{1}-x_{2}\right|\right) \\
\leq & \|v\|\|p\|_{0} \max \{2, b-a\}\left\|G_{0}\right\|_{2,0}\left|x_{1}-x_{2}\right| \\
& +\left\|G_{0}\right\|_{1,0}\|v\| \widetilde{\omega}_{p}\left(\left|x_{1}-x_{2}\right|\right) \\
& +\|v\|\|q\|_{0} \max \{2, b-a\}\left\|G_{0}\right\|_{2,0}\left|x_{1}-x_{2}\right| \\
& +\left\|G_{0}\right\|_{1,0}\|v\| \widetilde{\omega}_{q}\left(\left|x_{1}-x_{2}\right|\right) \\
\leq & \|v\| \omega\left(\left|x_{1}-x_{2}\right|\right),
\end{aligned}
$$

where

$$
\begin{aligned}
\omega(\delta) \equiv & \max \{2, b-a\}\left\|G_{0}\right\|_{2,0} \\
& \times\left\{\|p\|_{0} \delta+\|q\|_{0} \delta+\widetilde{\omega}_{p}(\delta)+\widetilde{\omega}_{q}(\delta)\right\} .
\end{aligned}
$$

By (7), the last inequality gives (41).

Assumption (iii) of Lemma 3.1 in [14] states that there exist positive constants $K^{\prime}$ and $K$, so that for any partition $\pi$,

$$
K^{\prime}\|v\| \leq\left\|v-T^{\prime} v\right\| \leq K\|v\|, \quad \forall v \in C_{\pi} .
$$

A proof of these inequalities is given in [14, pages 592-593], which takes the following outline in our case. Using (13), get

$$
\begin{aligned}
\| v & -T^{\prime}(v) \| \\
& =\left\|v-\int_{a}^{b}\left(p(\cdot) \partial_{x} G_{0}(\cdot, s)+q(\cdot) G_{0}(\cdot, s)\right) v(s) d s\right\| \\
& \leq\left\{1+\left\|G_{0}\right\|_{1,0}\left(\|p\|_{0}+\|q\|_{0}\right)(b-a)\right\}\|v\| .
\end{aligned}
$$

Similarly, using the representation

$$
\begin{aligned}
v= & v-T^{\prime}(v) \\
& +\int_{a}^{b}\left(p(\cdot) \partial_{x} G(\cdot, s)+q(\cdot) G(\cdot, s)\right)\left[v(s)-T^{\prime}(v)(s)\right] d s
\end{aligned}
$$

(see the first identity after (3.20) in [14]), obtain

$$
\|v\| \leq\left[1+\|G\|_{1,0}\left(\|p\|_{0}+\|q\|_{0}\right)(b-a)\right]\left\|v-T^{\prime}(v)\right\|,
$$

where $G$ is Green's function of IBVP (4a)-(4c).

Since all assumptions of Lemma 3.4 in [14] are satisfied, OSC problem (36) has a unique solution, and estimate (37) holds. Finally, estimate (38) follows from (37) and Jackson's theorem [19, Theorem 3.7.3].

Corollary 3. Let $u$ be the unique solution of problem (4a)(4c). Under the conditions of Lemma 2, OSC problem (18) has a unique solution $u_{h}$. If $u \in Q^{n+2}, n \leq 2$, then estimate (29) holds, and

$$
\left\|D^{r} u_{h}\right\| \leq C\left\|D^{r} u\right\|, \quad r \leq 2
$$

Proof. The solution $u_{h}$ of problem (18) is uniquely determined from the identity (35) with $g_{h}$ replaced by $y_{h}$, the unique solution of problem (36). Using $u_{h}^{\prime \prime}=y_{h}$, estimate (38) can be written in the form of (29) with $r=2$. Using (11) and (35), obtain

$$
D^{r}\left(u-u_{h}\right)=\int_{a}^{b} \partial_{x}^{r} G_{0}(x, s)\left(y-y_{h}\right)(s) d s, \quad r \leq 1 .
$$

Estimate (29) for $r \leq 1$ follows from the last identity and estimate (38). Using estimate (29) and the triangle inequality, obtain (49). 
Under sufficient regularity assumptions on the coefficients of the differential equation, in addition to the bounds in (49), higher order derivatives of the numerical solution $u_{h}$ are locally bounded by the corresponding derivatives of the exact solution $u$.

Lemma 4. Let $u$ and $u_{h}$ be the solutions of IBVP (4a)-(4c) and the OSC scheme (18), respectively, and let $n \geq 0$ be an integer. If $p, q \in Q^{2+n}$ and $u \in Q^{4+n}$, then

$$
\left\|D^{k} u_{h}\right\|_{C\left(I_{i}\right)} \leq C\left\|D^{k} u\right\|_{C\left(I_{i}\right)}, \quad k \leq 4+n, i=1, \ldots, N .
$$

Proof. The statement follows immediately from Lemma 4.1 in [14].

Proof of Theorem 1. By Corollary 3, the OSC problem (18) has a unique solution, and estimate (29) holds. Since functions $u$ and $u_{h}$ satisfy the same interface and boundary conditions, using Green's function $G$ of problem (4a)-(4c), we get

$$
\begin{aligned}
& D^{r} u(x)-D^{r} u_{h}(x) \\
&=\int_{a}^{b} \partial_{x}^{r} G(x, s)\left[(L u)(s)-\left(L u_{h}\right)(s)\right] d s, \\
& r \leq 1 .
\end{aligned}
$$

Let $Q: C[-1,1] \rightarrow P_{1}$ be the interpolation operator at the roots $\pm \sqrt{3} / 3$ of the Legendre polynomial of degree 2 . Let $Q_{\pi}: C[a, b] \rightarrow P_{1, \pi}$ be the piecewise interpolation operator at $\mathscr{G}_{h}$, the set of Gauss points, defined by

$$
\left.Q_{\pi} v\right|_{I_{i}}=S_{i}^{-1} Q S_{i} v
$$

where

$$
S_{i} v(s)=\widetilde{v}_{i}(s) \equiv v\left(x_{i+1 / 2}+\frac{s h_{i}}{2}\right) .
$$

Operator $Q_{\pi}$ is the so-called ssuper projector of order $(2, n)$, $n \leq 2$ [15, pages 22-23]. Since $Q_{\pi} L\left(u-u_{h}\right)=0$, rewrite

$$
L\left(u-u_{h}\right)=\left(1-Q_{\pi}\right) L u+\left(Q_{\pi}-1\right) L u_{h} .
$$

Note that

$$
\left.G(x, \cdot)\right|_{I_{i}} \in \begin{cases}C^{n}\left[t_{i-1}, t_{i}\right], & x \notin\left(t_{i-1}, t_{i}\right), \\ W^{1, \infty}\left(t_{i-1}, t_{i}\right), & x \in\left(t_{i-1}, t_{i}\right),\end{cases}
$$

where $W^{1, \infty}\left(t_{i-1}, t_{i}\right)$ is the Sobolev space. For $x \in \pi, r \leq 1$, and $n \leq 2$, obtain

$$
\begin{aligned}
& \left|\int_{a}^{b} \partial_{x}^{r} G(x, s)\left(1-Q_{\pi}\right) L u(s) d s\right| \\
& \quad \leq C h^{3+n} \sum_{i=1}^{N}\left\|\partial_{x}^{r} G(x, \cdot)\right\|_{C^{n}\left(I_{i}\right)}\|L u\|_{C^{2+n}\left(I_{i}\right)} \\
& \quad \leq C h^{3+n} N\left\|\partial_{x}^{r} G\right\|_{0, n}\left(\|u\|_{4+n}+\|p\|_{2+n}\|u\|_{3+n}+\|q\|_{2+n}\|u\|_{2+n}\right) \\
& \quad \leq C h^{2+n} \psi_{n, r},
\end{aligned}
$$

where $\psi_{n, r}$ is defined by (32). For $x \in\left(x_{j-1}, x_{j}\right), r \leq 1, n \leq 2$, and $n^{\prime}=\min \{n, 1-r\}$, obtain

$$
\begin{aligned}
& \left|\int_{a}^{b} \partial_{x}^{r} G(x, s)\left(1-Q_{\pi}\right) L u(s) d s\right| \\
& \leq C h^{3+n} \sum_{i=1, i \neq j}^{N}\left\|\partial_{x}^{r} G(x, \cdot)\right\|_{C^{n}\left(I_{i}\right)}\|L u\|_{C^{2+n}\left(I_{i}\right)} \\
& \quad+C h^{3+n^{\prime}}\left\|\partial_{x}^{r} G(x, \cdot)\right\|_{C^{n^{\prime}}\left(I_{j}\right)}\|L u\|_{C^{2+n^{\prime}}\left(I_{j}\right)} \\
& \leq C\left(h^{2+n}+h^{3+n^{\prime}}\right) \psi_{n, r} .
\end{aligned}
$$

Letting $r=1$ in the last estimate, obtain estimates of order 3 for both $n=1$ and $n=2$.

Similarly, using Lemma 4, obtain, for $r \leq 1$ and $n \leq 2$,

$$
\left|\int_{a}^{b} \partial_{x}^{r} G(x, s)\left(1-Q_{\pi}\right) L u_{h}(s) d s\right| \leq C \eta(h) \psi_{n, r},
$$

where

$$
\eta(h)= \begin{cases}h^{2+n}, & x \in \pi, \\ h^{2+n}+h^{3+\min \{n, 1-r\}}, & x \in[a, b] \backslash \pi .\end{cases}
$$

Using (52)-(59), obtain (30) and (31).

\section{Numerical Examples}

Example 1. Our first example is with a discontinuous coefficient in front of $u^{\prime \prime}$. We consider the BVP (4a)-(4c) in the form

$$
\begin{gathered}
k(x) u^{\prime \prime}=-\pi^{2} \sin \pi x, \quad x \in(0,0.5) \cup(0.5,1), \\
u(0)=u(1)=0, \quad[u]_{x=0.5}=0, \quad\left[k u^{\prime}\right]_{x=0.5}=1.8,
\end{gathered}
$$

where

$$
k(x)= \begin{cases}1, & x<0.5 \\ 0.1, & x \geq 0.5\end{cases}
$$

The exact solution of this problem is

$$
u(x)= \begin{cases}\sin \pi x, & x \in[0,0.5], \\ 10 \sin \pi x+18 x-18, & x \in[0.5,1] .\end{cases}
$$

Numerical results with the maximal nodal errors

$$
\left\|D^{r} e_{h}\right\|_{\pi}=\max \left\{\left|D^{r} u(x)-D^{r} u_{h}(x)\right|: x \in \pi\right\}, \quad r=0,1,
$$

the Sobolev norm errors, and the corresponding approximate convergence orders $p_{h}=\log _{2}\left(\left\|e_{h}\right\|_{*} /\left\|e_{h / 2}\right\|_{*}\right)$ are presented in Table 1. Observe fourth-order convergence of both the solution and its derivative in the maximal nodal norm. The latter demonstrates the superconvergence property of 
TABLE 1: The maximal nodal norm and the Sobolev norm errors and their approximate convergence orders for the problem in Example 1.

\begin{tabular}{|c|c|c|c|c|c|c|c|c|c|c|}
\hline$N$ & \multicolumn{2}{|c|}{$\left\|e_{h}\right\|_{\pi}$} & \multicolumn{2}{|c|}{$\left\|e_{h}^{\prime}\right\|_{\pi}$} & \multicolumn{2}{|c|}{$L^{2}$} & \multicolumn{2}{|c|}{$H^{1}$} & \multicolumn{2}{|c|}{$H^{2}$} \\
\hline 4 & $8.1-04$ & 3.4 & $7.3-03$ & 4.1 & $2.8-03$ & 4.1 & $4.4-02$ & 3.0 & $1.1+00$ & 2.0 \\
\hline 8 & $5.2-05$ & 4.0 & $4.5-04$ & 4.0 & $1.8-04$ & 4.0 & $5.5-03$ & 3.0 & $2.8-01$ & 2.0 \\
\hline 16 & $3.3-06$ & 4.0 & $2.8-05$ & 4.0 & $1.1-05$ & 4.0 & $6.9-04$ & 3.0 & $7.1-02$ & 2.0 \\
\hline 32 & $2.1-07$ & 4.0 & $1.7-06$ & 4.0 & $6.8-07$ & 4.0 & $8.6-05$ & 3.0 & $1.8-02$ & 2.0 \\
\hline 64 & $1.3-08$ & 4.0 & $1.1-07$ & 4.0 & $4.3-08$ & 4.0 & $1.1-05$ & 3.0 & $4.5-03$ & 2.0 \\
\hline 128 & $8.1-10$ & 4.0 & $6.8-09$ & 4.0 & $2.7-09$ & 4.0 & $1.3-06$ & 3.0 & $1.1-03$ & 2.0 \\
\hline 256 & $5.1-11$ & 4.0 & $4.2-10$ & 4.0 & $1.7-10$ & 4.0 & $1.7-07$ & 3.0 & $2.8-04$ & 2.0 \\
\hline 512 & $3.2-12$ & 4.0 & $2.6-11$ & 4.0 & $1.0-11$ & 4.0 & $2.1-08$ & 3.0 & $7.0-05$ & 2.0 \\
\hline 1024 & $2.0-13$ & 4.0 & $1.7-12$ & 4.0 & $6.6-13$ & 4.0 & $2.6-09$ & 3.0 & $1.7-05$ & 2.0 \\
\hline 2048 & $9.1-15$ & 4.4 & $1.4-13$ & 3.6 & $4.5-14$ & 3.9 & $3.3-10$ & 3.0 & $4.3-06$ & 2.0 \\
\hline
\end{tabular}

TABLE 2: The maximal nodal norm and the Sobolev norm errors and their convergence orders for the problem in Example 2.

\begin{tabular}{|c|c|c|c|c|c|c|c|c|c|c|}
\hline$N$ & \multicolumn{2}{|c|}{$\left\|e_{h}\right\|_{\pi}$} & \multicolumn{2}{|c|}{$\left\|e_{h}^{\prime}\right\|_{\pi}$} & \multicolumn{2}{|c|}{$L^{2}$} & \multicolumn{2}{|c|}{$H^{1}$} & \multicolumn{2}{|c|}{$H^{2}$} \\
\hline 4 & $2.1-07$ & 4.0 & $2.6-06$ & 4.0 & $4.3-06$ & 4.0 & $5.9-05$ & 3.0 & $1.5-03$ & 2.0 \\
\hline 8 & $1.3-08$ & 4.0 & $1.6-07$ & 4.0 & $2.7-07$ & 4.0 & $7.4-06$ & 3.0 & $3.8-04$ & 2.0 \\
\hline 16 & $8.2-10$ & 4.0 & $1.0-08$ & 4.0 & $1.7-08$ & 4.0 & $9.2-07$ & 3.0 & $9.6-05$ & 2.0 \\
\hline 32 & $5.2-11$ & 4.0 & $6.4-10$ & 4.0 & $1.1-09$ & 4.0 & $1.2-07$ & 3.0 & $2.4-05$ & 2.0 \\
\hline 64 & $3.2-12$ & 4.0 & $4.0-11$ & 4.0 & $6.7-11$ & 4.0 & $1.4-08$ & 3.0 & $6.0-06$ & 2.0 \\
\hline 128 & $2.0-13$ & 4.0 & $2.5-12$ & 4.0 & $4.2-12$ & 4.0 & $1.8-09$ & 3.0 & $1.5-06$ & 2.0 \\
\hline 256 & $1.3-14$ & 4.0 & $1.6-13$ & 4.0 & $2.6-13$ & 4.0 & $2.3-10$ & 3.0 & $3.7-07$ & 2.0 \\
\hline 512 & $1.9-15$ & 2.8 & $9.5-15$ & 4.0 & $1.7-14$ & 4.0 & $2.8-11$ & 3.0 & $9.3-08$ & 2.0 \\
\hline
\end{tabular}

the OSC solution. The convergence in the Sobolev $H^{k}$-norm is of order $h^{4-k}$ for $k=0,1,2$ which are of optimal order for the finite element space of piecewise Hermite cubics. The error estimates in the maximum norm are similar to those in the Sobolev norms. The machine precision is reached at $N=2048$. The numerical results are consistent with the theoretical estimates given in Theorem 1 .

Example 2. We now apply the OSC scheme to the problem in Example 1 of [7, page 604] formulated as follows:

$$
\begin{gathered}
u^{\prime \prime}=f \quad \text { in }(0,0.5) \cup(0.5,1), \\
u(0)=\alpha, \quad u(1)=\beta, \quad[u]=0, \quad\left[u^{\prime}\right]=1,
\end{gathered}
$$

with the exact solution

$$
u(x)=\exp \left(-\frac{x}{\epsilon}\right)-0.5 \begin{cases}x, & x \leq 0.5 \\ 1-x, & x \geq 0.5\end{cases}
$$

For the case $\epsilon=1$ and $c=0.5$, numerical results are given in Table 2, and they are qualitatively similar to those in the preceding example. The machine precision is now reached at $N=512$.

\section{Conclusions}

The OSC method applied to IBVP (4a)-(4c) provides a highly accurate numerical solution to a problem with discontinuous coefficients. The scheme preserves the advantages of the OSC method applied to BVPs with smooth solutions. The resulting coefficient matrix has the same structure as that of the corresponding BVP without interface conditions. The obtained results can be extended to multiple interface points and to corresponding nonlinear and time dependent problems. They may also be helpful in developing OSC schemes for multidimensional interface problems.

\section{Conflict of Interests}

The authors declare that there is no conflict of interests regarding the publication of this paper.

\section{References}

[1] R. Aitbayev, "Existence and uniqueness for a two-point interface boundary value problem," Electronic Journal of Differential Equations, vol. 2013, no. 242, pp. 1-12, 2013.

[2] C.-K. Chen, "A fixed interface boundary value problem for differential equations: a problem arising from population genetics," Dynamics of Partial Differential Equations, vol. 3, no. 3, pp. 199-208, 2006.

[3] M. Mikhailov and M. Ozisik, Unified Analysis and Solutions of Heat and Mass Diffusion, Dover, New York, NY, USA, 1994.

[4] A. N. Tikhonov and A. A. Samarskii, "Homogeneous difference schemes," USSR Computational Mathematics and Mathematical Physics, vol. 1, pp. 5-67, 1962.

[5] W. Welsh and T. Ojika, "Multipoint boundary value problems with discontinuities. I. Algorithms and applications," Journal of Computational and Applied Mathematics, vol. 6, no. 2, pp. 133143, 1980. 
[6] C. S. Peskin, "The immersed boundary method," Acta Numerica, vol. 11, pp. 479-517, 2002.

[7] H. Huang and Z. Li, "Convergence analysis of the immersed interface method," IMA Journal of Numerical Analysis, vol. 19, no. 4, pp. 583-608, 1999.

[8] Z. Li, "The immersed interface method using a finite element formulation," Applied Numerical Mathematics, vol. 27, no. 3, pp. 253-267, 1998.

[9] Z. Li and K. Ito, The Immersed Interface Method: Numerical Solutions of PDEs Involving Interfaces and Irregular Domains, Frontiers in Applied Mathematics, SIAM, 2006.

[10] X.-D. Liu and T. C. Sideris, "Convergence of the ghost fluid method for elliptic equations with interfaces," Mathematics of Computation, vol. 72, no. 244, pp. 1731-1746, 2003.

[11] A. Loubenets, T. Ali, and M. Hanke, "Highly accurate finite element method for one-dimensional elliptic interface problems," Applied Numerical Mathematics, vol. 59, no. 1, pp. 119-134, 2009.

[12] U. M. Ascher, R. M. Mattheij, and R. D. Russell, Numerical Solution of Boundary Value Problems for Ordinary Differential Equations, SIAM, Philadelphia, Pa, USA, 1995.

[13] G. Fairweather and D. Meade, "A survey of spline collocation methods for the numerical solution of differential equations," in Mathematics for Large Scale Computing, vol. 120, pp. 297-341, Marcel Dekker, New York, NY, USA, 1989.

[14] C. de Boor and B. Swartz, "Collocation at Gaussian points," SIAM Journal on Numerical Analysis, vol. 10, pp. 582-606, 1973.

[15] C. de Boor and B. Swartz, "Local piecewise polynomial projection methods for an O.D.E. which give high-order convergence at knots," Mathematics of Computation, vol. 36, no. 153, pp. 2133, 1981.

[16] T. J. Pignani and W. M. Whyburn, "Differential systems with interface and general boundary conditions," Journal of the Elisha Mitchell Scientific Society, vol. 72, pp. 1-14, 1956.

[17] M. H. Schultz, Spline Analysis, Prentice-Hall, Englewood Cliffs, NJ, USA, 1973.

[18] G. Fairweather and I. Gladwell, "Algorithms for almost block diagonal linear systems," SIAM Review, vol. 46, no. 1, pp. 49-58, 2004.

[19] K. Atkinson and W. Han, Theoretical Numerical Analysis: A Functional Analysis Framework, Springer, New York, NY, USA, 2009. 


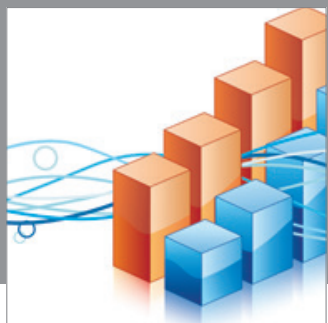

Advances in

Operations Research

mansans

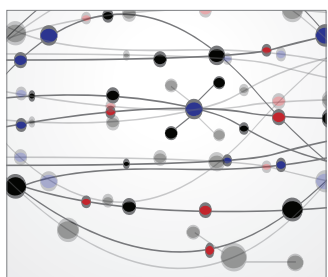

The Scientific World Journal
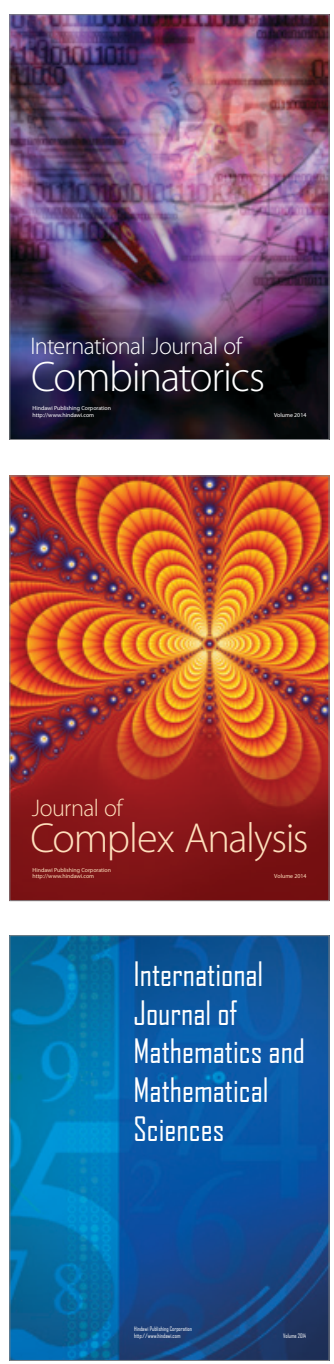
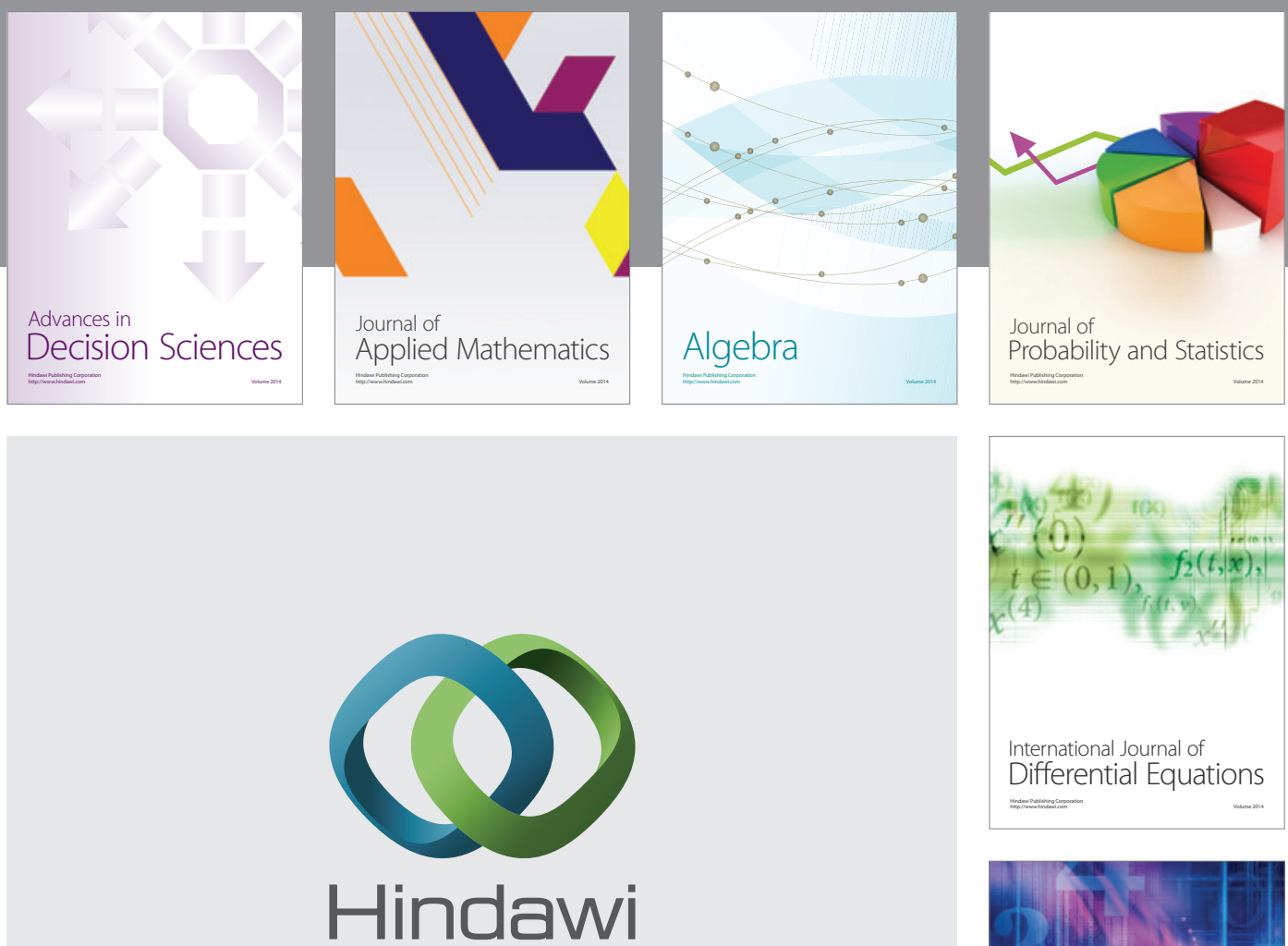

Submit your manuscripts at http://www.hindawi.com
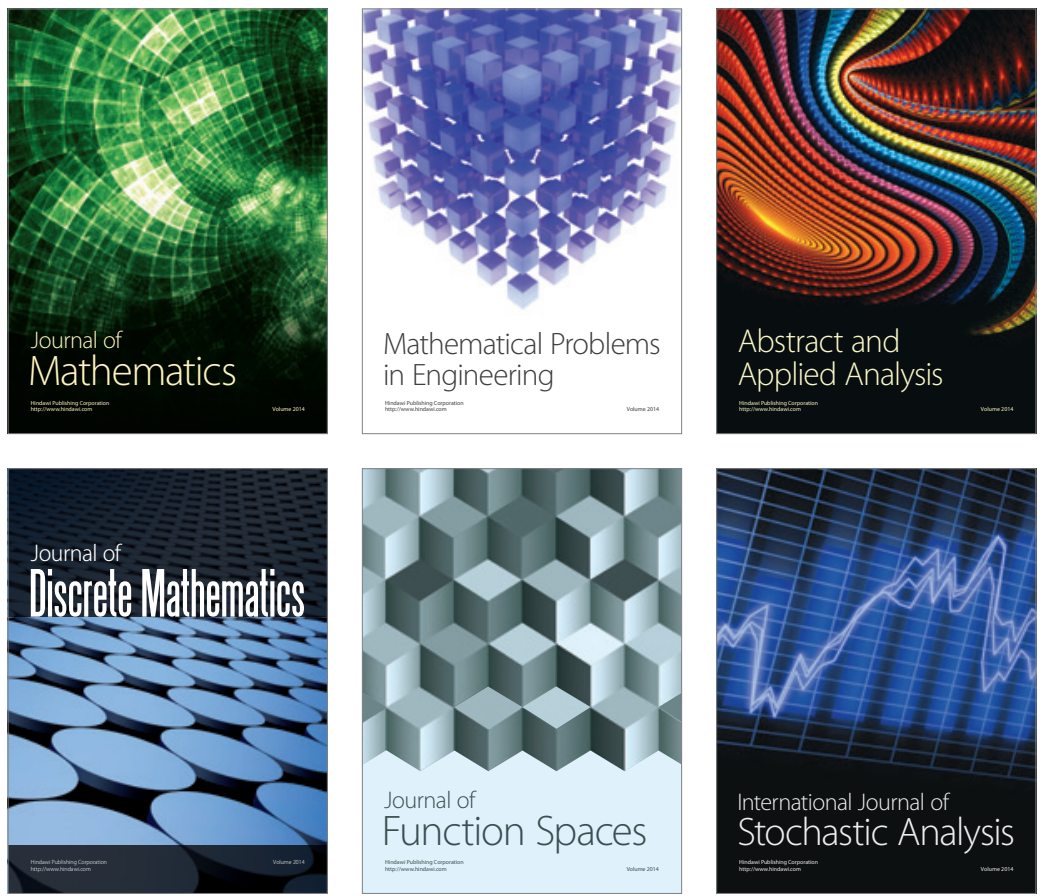

Journal of

Function Spaces

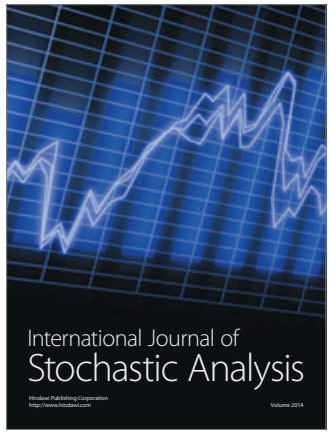

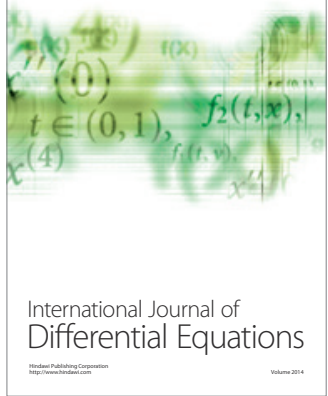
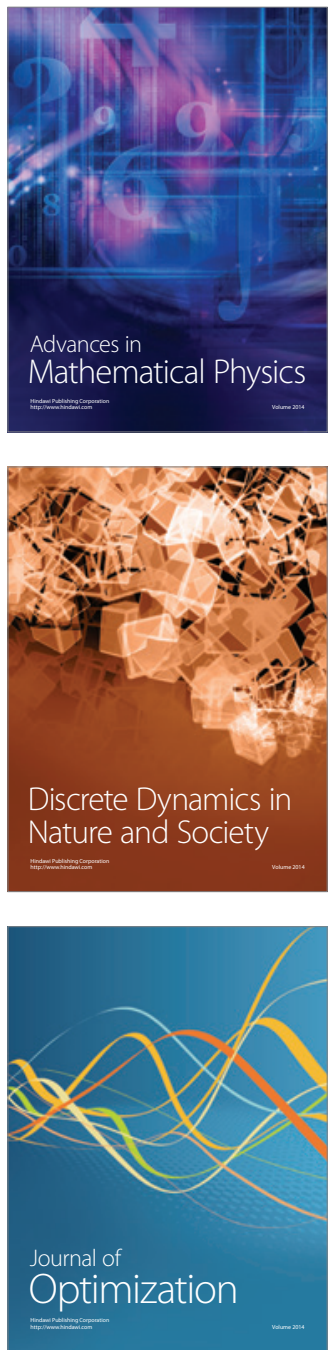